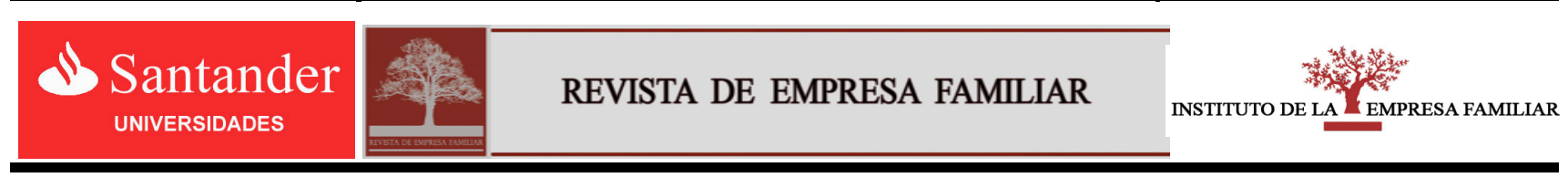

www.revistadeempresafamiliar.uma.es

\title{
Family office: instrumento de gestión del patrimonio familiar
}

\author{
Family office: wealth management in the family context \\ Elena Rivo López ${ }^{\mathrm{a},} \cdot$ Beatriz González Vázquez $^{\mathrm{b}} \cdot$ Nuria Rodríguez López $^{\mathrm{c}}$ \\ ${ }^{a, b, c}$ Departamento de Organización de Empresas y Marketing. Universidad de Vigo, 36310 (Spain)
}

\author{
D A T OS A R T Í C U L O \\ Historial: \\ Recibido 21-06-2010 \\ Aceptado 02-11-2011 \\ Palabras clave: \\ Family Office \\ Patrimonio Familiar \\ Estrategia \\ Inversión \\ Códigos JEL: \\ K36
}

\section{A R T I C L E IN F O \\ Article history: \\ Received 21 June 2010 \\ Accepted 02 November 2011}

Keywords:

Family Office

Family wealth

Strategy

investment

JEL codes:

$\mathrm{K} 36$
R E S U M E N

Este trabajo estudia el concepto de Family Office, considerándola como un instrumento más que puede utilizar la familia empresaria para gestionar y diversificar sus activos $\mathrm{y}$ riesgos. Trataremos de profundizar en las diferentes estrategias que se pueden seguir con objetivos diferentes según la modalidad de "family office" implementada, así como identificar las características diferenciadoras entre las diversas estructuras existentes. El concepto tradicional de family office (FO) se centra en un negocio dirigido por y para una sola familia. Su principal función consiste en centralizar la gestión de un patrimonio familiar significativo. El objetivo es la transferencia del patrimonio entre generaciones.

A B S T R A C T

This paper studies the concept of Family Office, as a tool available to the members of family business to manage and diversify their assets and risks. First, the research makes a theoretical analysis of the concept, the different modalities and different ways of implementing a family office. Then, this study analyzes the different strategies that can be taken with different purposes (depending on the type of family office implemented), and the differential characteristics between the several structures. The traditional concept of family office $(F O)$ focuses on a business run by and for one family. Its function is to centralize the management of a significant family fortune. Typically, these organizations employ staff to manage investments, taxes, philanthropy, trusts and legal services. The aim is to transfer wealth between generations.

\footnotetext{
Autor de contacto. Tel.: +34988368734

Correoselectrónicos: rivo@uvigo.es,bgonza@uvigo.es,nrl@uvigo.es
} 


\section{Introducción}

Una family office (FO) es una empresa privada que gestiona inversiones y fideicomisos para una familia (Amit, Liechtenstein y Prats, 2009). El capital financiero de la empresa es el propio capital de la familia, a menudo acumulado durante generaciones por ésta. Las family offices pueden dedicarse desde la tradicional prestación de servicios personales, tales como la gestión del personal doméstico y la gestión de viajes, hasta la realización de inversiones de capital riesgo o capital desarrollo. Dado el elevado coste operativo de una family office, se considera que resulta interesante la creación de este tipo de estructuras a partir de patrimonios superiores a 100 millones de dólares -64, 7 millones de euros- (Casado, Martínez, López, Butler, Sterba, Cazorla y Prado, 2008).

$\mathrm{Si}$ bien, existen diferencias por zonas geográficas ya que, como se aprecia en la tabla 1, mientras en Europa encontramos más $F O$ en familias con activos superiores al billón de dólares, en América predominan las familias con patrimonio comprendido en el tramo de 100-500 millones de dólares (véase tabla 1). En España, la mayoría de las $F O$ se crean a partir de activos comprendidos entre 25 y 30 millones de euros. Se calcula que su coste operativo supone aproximadamente un $0,5 \%$ del patrimonio.

\section{Tabla 1}

Patrimonio familiar. Comparación del caso europeo y americano.

\begin{tabular}{lll}
\hline $\begin{array}{l}\text { Patrimonio } \\
\text { familiar: caso } \\
\text { americano } \\
\text { Frecuencia (\%) }\end{array}$ & $\begin{array}{l}\text { Patrimonio } \\
\text { familiar: caso } \\
\text { europeo } \\
\text { Frecuencia }(\%)\end{array}$ & $\begin{array}{l}\text { Valor } \\
\text { monetario }(\$)\end{array}$ \\
\hline $52 \%$ & $30 \%$ & $\begin{array}{l}100 \mathrm{~m} . \$- \\
500 \mathrm{~m} . \$\end{array}$ \\
$17 \%$ & $11 \%$ & $500 \mathrm{~m} . \$-$ \\
$26 \%$ & $53 \%$ & $1 \mathrm{bn} . \$$ \\
$5 \%$ & $6 \%$ & $>1 \mathrm{bn} . \$$ \\
\hline
\end{tabular}

Fuente: elaboración propia a partir de Amit, Liechtenstein, y Prats (2009)
El concepto tradicional de family office se centra en un negocio dirigido por y para una sola familia. $\mathrm{Su}$ principal función consiste en centralizar la gestión de un patrimonio familiar significativo.

Normalmente, estas organizaciones emplean a personal para gestionar las inversiones, impuestos, actividades filantrópicas, fideicomisos y asuntos legales. El objetivo es la transferencia de riqueza entre generaciones. La $F O$ invierte el dinero de la familia, gestiona todos sus activos, y desembolsa los pagos a los miembros de la familia según sea necesario.

En la actualidad, se calcula que pueden existir entre unas 70 u $80 F O$ en España, lo que supone entre un $10 \%$ y un $11 \%$ de las que existen en Europa (Casado, Vidal-Rivas, Brito, Salgado, Verdaguer, Willette y Pastor, 2009).

Las $F O$ se perfilan como gestoras de las inversiones que realiza una familia empresaria, con el fin de asegurar el patrimonio para las siguientes generaciones. Este tipo de estructuras no han sido ampliamente estudiadas, ni tampoco ampliamente implantadas en España. Por este motivo nos resulta interesante como objeto de investigación, recurriendo al estudio de algunos ejemplos que encontramos en nuestro país, puesto que la documentación teórica es escasa y en general referida a Estados Unidos.

\section{Evolución histórica de las Family office}

La literatura sobre la moderna $F O$ es escasa, e incluso menor para el caso de FO de una única familia. La razón radica sobre todo en el hecho de que las familias de elevado nivel económico quieren privacidad. Otra motivación se basa en que, dependiendo de cómo se defina la $F O$, ésta puede abarcar una multitud de estructuras, que van desde un miembro de la familia haciendo tareas administrativas para su familia junto a otras tareas en una empresa familiar, hasta un equipo de profesionales enfocados en la inversión, la contabilidad, asuntos jurídicos, y servicios personales (Avery, 2004; Martiros y Millay, 2006). Servicios personales que pueden incluir actividades tales como la gestión de viajes, o la gestión del personal doméstico, entre otros. 
Entre las diversas definiciones propuestas por los profesionales destacaríamos: una organización para apoyar las necesidades financieras de una familia específica (a partir de la asignación de activos estratégicos para el mantenimiento de registros y presentación de informes) (Wolosky, 2002); un centro de influencia y estabilidad para ayudar a familias muy ricas a garantizar la conservación y el crecimiento de sus activos financieros y el patrimonio familiar (Avery, 2004); y una estructura creada para gestionar los activos de una familia acaudalada (Curtis, 2001).

Sobre esta base teórica y buscando un término con amplitud y sencillez conceptual, se ha adoptado la siguiente definición de FO: un centro de profesionales dedicados a atender las necesidades financieras y personales de una familia con patrimonio elevado (Amit, 2006).

\section{Razones para crear una Family Office}

El crecimiento de una empresa familiar hace que el responsable familde la gestión se enfrente a un doble reto: la gestión de la empresa familiar, y la gestión de los servicios a la Familia y del patrimonio que ha logrado acumular a lo largo de los años. Inicialmente suele buscarse ayuda especializada para la gestión de activos, fundamentalmente en áreas como la contabilidad y el mantenimiento de registros (Wolosky, 2002). Según Avery (2004), la necesidad de ayuda externa se acentúa generalmente ante la venta de la empresa familiar y la liquidación repentina de una inmensa cantidad de dinero. La segunda $y$ subsiguientes generaciones, que heredan grandes fortunas o las adquieren repentinamente tras la venta del negocio familiar, a menudo carecen de tiempo y experiencia para gestionar sus activos adecuadamente (Rivo, Rodríguez y González, 2011).

Ante esta situación deberían analizarse las razones que llevarían a una familia a elegir una family office en lugar de otra forma de optimización de la riqueza. Curtis (2001) cita a un gerente de $F O$ : "La razón principal tiene que ver con el desafío de la administración: nadie va a tomar tan en serio sus problemas como los tomaría uno mismo." De hecho, estudios previos sugieren que el servicio personalizado, la confidencialidad, el control y la flexibilidad son algunos de los principales beneficios citados por las familias que poseen una family office en funcionamiento (Avery, 2004). La FO suele ser tratada casi como parte de la familia (Avery, 2004; Newton, 2002) y considerada como el mejor medio de preservar la riqueza transgeneracional (Avery, 2004). Otros factores clave que recomiendan la creación de las $F O$ frente a otro tipo de estructuras son la privacidad, la ausencia de conflictos de intereses, la estructura flexible, la exclusividad y la discreción (Allen, 2007).

Según Gray (2004), aumentar la felicidad, mejorar el estilo de vida de los miembros de la familia, y proporcionar un liderazgo familiar para preparar a la próxima generación en sus responsabilidades, son criterios válidos e importantes, aunque no comerciales, para el éxito en un contexto de $F O$.

Siguiendo con el análisis de las posibles motivaciones para crear una $F O$, nos encontramos con un mayor control como una de las principales razones citadas por las familias. Sin embargo, si se pregunta a gerentes (no familiares) de $F O$ por sus prioridades, el control no es ni siquiera un objetivo planteable; para ellos, la generación de beneficios es su meta primordial (Rivo, Rodríguez y González, 2011).

Esta variedad de motivaciones que justifican la puesta en marcha de una $F O$ serán decisivas a la hora de determinar los servicios prestados, los criterios de selección de personal (tanto interno como subcontratado), los mecanismos de gestión, medición del rendimiento, y visión de futuro para el mercado de $F O$.

La literatura refleja esta diversidad. La mayoría de artículos se refieren a las distintas alternativas para la gestión de un gran patrimonio, entre ellos diferentes tipos de proveedores de servicios de $F O$, en lugar de concentrarse en un tipo específico (Newton, 2002; Shaw-Grove y Prince, 2004; Gray, 2005; Avery, 2004; Wolosky, 2002; Gray, 2004). También debe mencionarse que en los últimos 
años las pequeñas firmas que se especializaron en un servicio concreto han sido adquiridas por grandes empresas que quieren ofrecer una gama completa de productos a las familias (Rivo, Rodríguez y González, 2011).

Los estudios reflejan claramente por qué cada vez más se utilizan $F O$, siendo las razones más importantes, la privacidad, el control, la flexibilidad y el servicio individualizado. Sin embargo, falta en la literatura una guía sobre cómo evaluar el rendimiento de la $F O$. De hecho, la investigación preliminar (Martiros, y Millay, 2006) sugiere que la $F O$ está buscando foros de conocimiento que proporcionen una normalización de su gestión así como más transparencia de mercado, a fin de garantizar una experiencia más coherente y eficaz. Otro gap de conocimientos lo encontramos a la hora de investigar sobre las características diferenciadoras entre las $F O$ que se encuentran en funcionamiento en la actualidad.

\section{Tipos de Family Office}

El diseño y configuración de una $F O$ responde a la cultura de valores de cada familia. Ésta fijará los objetivos que pretende alcanzar con la creación y desarrollo de una $F O$, determinando los tipos de actividad y activos que conformarán el esqueleto de este tipo de estructura. Esto implica una enorme dificultad a la hora de establecer agrupamientos y características diferenciadoras entre distintas FOs (Rivo, Rodríguez y González, 2011).

En España, el embrión a una $F O$ parte de la consideración de que en las compañías siempre ha existido la figura de un director financiero o de un director administrativo que se ha dedicado a gestionar no sólo los negocios de la empresa, sino también a encargarse de los temas particulares de los miembros de la familia; la motivación reside en el hecho de que el empresario o el fundador se ha dedicado exclusivamente al trabajo, casi sin tiempo de poderle prestar cierta atención a la mejora de la gestión patrimonial.

En este contexto español se podrían identificar tres tipos de family office (Casado, Martínez, López, Butler, Sterba, Cazorla y
Prado, 2008). Denominaremos FO Tipo 1, aquella family office que ofrece servicios exclusivamente a los accionistas que forman parte de la empresa familiar. Estos servicios son de diversa índole: desde ayudarles a afrontar sus viajes, a legalizar a las personas que realizan tareas domésticas en sus casas, como a ayudarles a gestionar su patrimonio (gestión de inmuebles, gestión de inversiones en Bolsa, etc.) pero que no realicen inversiones como empresa de capital desarrollo o de capital riesgo (Rivo, Rodríguez y González, 2011).

Existe otro tipo de family office (Tipo 2) que además de proveer todos estos servicios a los miembros de la familia, realiza inversiones de capital de riesgo o de capital desarrollo.

Un tercer tipo de family office (Tipo 3) es aquel que conforma puramente una empresa de inversión (cuya denominación anglosajona se corresponde con Investment Conference), bien sean en capital riesgo, en capital de desarrollo, etc. Este tipo de $F O$ puede tener dos orígenes: uno, que es posiblemente el más conocido, es el que proviene de la desinversión de la propia empresa familiar. Es decir, se vende la empresa familiar o sus participaciones. En definitiva, se trata de miembros de la empresa que quieren continuar unidos a la familia y constituyen una $F O$ para canalizar su capital en distintas inversiones (Tipo 3 desinversión). El otro posible origen supone la creación de $F O$, provenientes de empresas familiares, que además de tener su gestión de familia por un lado, constituyen una empresa puramente de inversión para diversificar patrimonio que proviene de una empresa familiar, o ya provenía de otro tipo (Tipo 3 diversificación).

En la tabla 2 se muestran algunas de las $F O$ creadas en España (en funcionamiento en el año 2010), la modalidad a la que pertenecen, la familia propietaria y la empresa familiar de origen.

Estudios de investigación realizados sobre empresas reales ya en funcionamiento en Europa y América, distinguen cinco posibles casos de family office (Amit, Liechtenstein, y 
Prats, 2009) ${ }^{1}$, que se podrían englobar perfectamente dentro de las tres modalidades que acabamos de ver para el contexto exclusivamente español. Dichos estudios han sido realizados por la Wharton University of Pennsylvania, en colaboración con el IESE, analizando una muestra de $\mathrm{F}$ que manejan fondos superiores a 100 millones de dólares (64,7 millones de euros) invertidos en activos repartidos por todo el mundo; la investigación se basa en entrevistas realizadas a miembros destacados de estas organizaciones. la gestión de inversiones es externalizada. El responsable de la $F O$ dedica su tiempo a atender a los distintos miembros de la familia. Se trabaja con tres generaciones distintas con necesidades y objetivos diferentes. Mientras algunos miembros de la familia solicitan un crecimiento agresivo, otros prefieren un enfoque centrado en la preservación del capital. Los miembros de la $F O$ supervisan las inversiones y asesoran a los familiares (Rivo, Rodríguez y González, 2011).

Aproximadamente una quinta parte de su riqueza total la invierten principalmente en renta fija, renta variable e inmuebles. El resto está

\section{Tabla 2}

Tipología y ejemplos de Family Office en España.

\begin{tabular}{llll}
\hline Modalidad FO & Family Office & Familia & Empresa familiar \\
\hline TIPO 1: SERVICIOS & Corporación Exea & Familia Puig & Grupo Puig \\
\hline $\begin{array}{l}\text { TIPO 2: } \\
\text { Servicios+inversión }\end{array}$ & Grupo Landon & Familia Gallardo & Almirall Prodesfarma \\
& $\begin{array}{l}\text { Casa Grande de Cartagena } \\
\text { Lafont }\end{array}$ & $\begin{array}{l}\text { Familia Del Pino } \\
\text { Familia Carulla }\end{array}$ & $\begin{array}{l}\text { Ferrovial } \\
\text { Agrolimen }\end{array}$ \\
\hline $\begin{array}{l}\text { TIPO 3: Inversión } \\
\text { Origen desinversión } \\
\text { familiar }\end{array}$ & Omega Capital & Familia Koplowitz & Venta FCC \\
& Caboel & Familias Carbó, Botet & Venta Caprabo \\
& Fundación Numa & y Elías & \\
& Nefinsa & Familia Sanz-Gras & Venta Dalphimetal \\
& Bernat Family Office (BFO) & Familia Serratosa & Venta Gamesa \\
& & & Venta Chupa Chups \\
\hline TIPO 3: Inversión & Grupo Quercus & Familia Carulla & Agrolimen \\
$\begin{array}{l}\text { Diversificación } \\
\text { patrimonio }\end{array}$ & Hemisferio & Familia Lara & Grupo Planeta \\
& Libertas 7 & Familia Noguera & Banco de Valencia \\
\hline
\end{tabular}

Fuente: elaboración propia

Dentro del primer tipo, $F O$ dedicada exclusivamente a la prestación de servicios, incluimos el caso 2 del citado estudio.

CASO 2: Family office multigeneracional, orientada a la prestación de servicios.

Creadas hace casi treinta años, se basan en la gestión fiscal. Suelen estar formadas por 15 profesionales inversores y varios abogados, contables, economistas y administrativos. Toda

\footnotetext{
${ }^{1}$ Un estudio piloto realizado por la Wharton University of Pennsylvania en colaboración con el IESE, analiza una muestra de family office que manejan fondos superiores a 100 millones de dólares (64,7 millones de euros) invertidos en activos repartidos por todo el mundo; la investigación se basa en entrevistas realizadas a miembros destacados de
} estas organizaciones.

Rivo, E., González-Vázquez, B. y Rodríguez López, N. (2011). Family office: un instrumento de gestión del patrimonio en la Empresa Familiar. Revista de Empresa Familiar, 1(2), 43-57. incorporado en la empresa familiar en cuya gestión están implicadas muy directamente dos generaciones.

En la segunda modalidad, $F O$ que combina la prestación de servicios y actividades de inversión, englobaríamos los casos 3 y 5.

CASO 3: Family office enfocada en la preservación de la riqueza.

Abarca varias generaciones, varios países y alrededor de 15 beneficiarios. Esta $F O$ ha sido encargada de lograr la unidad familiar y una inteligente y ordenada sucesión del patrimonio. Se creó hace unos 20 años. Ayuda a la familia en tareas administrativas tales como la banca, 
los impuestos y la presentación de informes, así como servicios de conserjería tipo $y$ planificación del patrimonio. La mayoría de la inversión se realiza en bienes inmuebles a través de inversores externos. El responsable de la $F O$ es un miembro de la familia cuya fortaleza consiste en conocer y comprender a los miembros de la familia y manejar astutamente a los asesores financieros externos.

CASO 5: Family Office enfocada en la evolución a largo plazo.

En la década de los setenta, el término $F O$ no existía, sin embargo sí funcionaban como tal algunas empresas. La familia utilizaba los empleados disponibles en su empresa familiar (secretarias, contables, tesorero, abogados) con tal fin. Los cambios en las leyes fiscales, cada vez más complejas, implicaron la necesidad de planificación de bienes y el deseo de transmitir a la siguiente generación el patrimonio generado, supuso una demanda de contratación de expertos en la materia. Además, la familia también quería diversificar sus inversiones en sectores con los que no estaba familiarizada, comenzando así a contratar profesionales con experiencia en esas áreas.

En la década de los ochenta, la familia comenzó a ver el lado negativo de tener un gran equipo de expertos en la empresa, puesto que si a largo plazo se obtuviesen resultados negativos, tendría que replantearse la continuidad del equipo inversor (pero $\sin$ posibilidad de rectificación en los resultados obtenidos).

En los noventa es cuando se plantea la creación de una $F O$, con participación de expertos profesionales externos. Un directivo de esta $F O$ considera que una $F O$ de primera generación será muy diferente a la de una tercera o cuarta generación puesto que los objetivos de cada una de ellas son muy distintos (Amit, Liechtenstein, yPrats, 2009). Las próximas generaciones están interesadas en la forma en que se están manejando sus inversiones y sus efectos fiscales.

Se evalúa el éxito de la $F O$ con dos criterios: en primer lugar, las inversiones que deben estar adecuadamente diversificadas $y$ en segundo lugar, la atención a las necesidades de la familia (inversiones, necesidades de liquidez, formación para la generación más joven, servicios de conserjería, seguros, asesoría fiscal y jurídica, actividades filantrópicas, y asegurar que la familia no sea expuesta a riesgos excesivos).

Esta $F O$ contrata a un profesional con perfil generalista, cuya responsabilidad es coordinar una serie de expertos externos que satisfagan las necesidades familiares. La familia sigue una estrategia de inversión conservadora cuya prioridad principal es conservar el patrimonio frente a un crecimiento agresivo pero más arriesgado. A través de programas de formación, se trata de transmitir esta idea a la siguiente generación.

Por último, en la tercera modalidad, dentro de las $F O$ enfocadas únicamente a la inversión se incluirían el caso 1 y 4 .

CASO 1: Family office dedicada exclusivamente a la inversión.

Se trata de las $F O$ más grandes y más profesionales, creadas hace menos de una década. Se trata de una compañía de inversión privada no con objetivos fiscales sino centrada en objetivos financieros trabajando con los principales expertos en inversión. Su objetivo principal consiste en lograr el mayor crecimiento posible del patrimonio familiar. Se trata de lograr que los miembros de la familia puedan dedicar tiempo a sus propios negocios. El responsable de la $F O$ suele tener experiencia en banca de inversión y su equipo incluye a nueve inversores profesionales (entre analistas y traders), 10 contables, un abogado y una plantilla administrativa de 12 personas.

\section{CASO 4: Family office dedicada a la inversión.}

$\mathrm{Su}$ función principal es la inversión, pero entendida ésta como algo más que simplemente invertir en Bolsa. Las inversiones se realizan con el objetivo de preservar el patrimonio además de generar un retorno anual positivo, fijado por el equipo directivo de esta $F O$ en un rendimiento al menos un 2\% superior al SyP 500 (Amit, Liechtenstein, yPrats, 2009). Otras funciones desempeñadas por la $F O$ incluyen la coordinación de asistencia jurídica, fiscal y de 
planificación de sucesión, y la supervisión de actividades filantrópicas.

Este tipo de $F O$ emplea a un joven y dinámico jefe de inversión que mantiene una relación estrecha y directa con el responsable del $F O$. Él coordina las inversiones y mantiene las relaciones con los expertos externos a la empresa. Se externaliza la asistencia jurídica, la fiscal, el cuidado de los inmuebles, existiendo un director encargado de la colección de arte. Se trata de reducir al mínimo la burocracia y externalizar actividades siempre que sea posible.

El responsable de la $F O$ y su equipo se reúnen una vez por semana para discutir sobre temas como inversiones específicas, carteras de confianza, la estrategia general de inversión, informes de analistas o la asignación de capital.

El máximo ejecutivo de esta $F O$ analizada considera que una buena relación entre los profesionales y la familia es un factor clave de éxito para una family offic (Amit, Liechtenstein, yPrats, 2009). Los gestores consideran que la $F O$ debería reflejar las circunstancias particulares y la personalidad de la familia que la crea.

\section{Implantación de una Family Office}

Cada $F O$ reflejará los valores y objetivos de cada familia, incidiendo este hecho lógicamente en los tipos de actividad y activos que conformen esta estructura.

Consecuentemente, a la hora de crear una $F O$, lo primero que la familia debe plantearse es qué objetivo u objetivos pretende alcanzar a través de la misma (objetivos familiares, como la transmisión del legado de principios y valores familiares a través de las distintas generaciones; objetivos patrimoniales como pueden ser el mantenimiento y transmisión de la riqueza, su crecimiento, un crecimiento más agresivo,...). Una vez determinados, tendrá que pensar qué servicios o actividades habría que llevar a cabo para conseguirlos. Por último, deberá decidir cuál debe ser la estructura organizativa que permita lograr las citadas metas (incluyendo la decisión de realizar las actividades con personal interno o externo).

Una $F O$ puede dedicarse a la prestación de servicios a los miembros de la familia empresaria, a la realización de servicios más actividades de inversión o exclusivamente a actividades de inversión.

Se pueden mencionar como posibles actividades a realizar desde una FO (Martin, Lansberg, y Darst, 1999) las inversiones en activos, la asignación de activos (allocation), servicios fiscales, servicios bancarios, asesoramiento legal, gestión de seguros, servicios personales, actividades filantrópicas, formación y educación. Estas actividades pueden ser realizadas en su totalidad por los miembros de la $F O$, pueden ser externalizadas o una combinación de ambas, que suele ser la situación más frecuente.

Existen dos tipos de estructuras que ofrecen servicios profesionales familiares. En la primera, la familia tiene su propia $F O$ (Single Family Office), lo que garantiza la individualidad y una total confidencialidad. El segundo tipo se trataría de $F O$ que sirven a más de una familia (entre cinco o seis familias, Multy Family Office). Obviamente, las familias saben quiénes son los otros clientes con los que comparte los mismos servicios de oficina. Esta modalidad tiene escasa aplicación todavía en España, pero sí es de común utilización en otros países como por ejemplo en EE.UU.

El tamaño de las $F O$ puede ser muy diverso. En España suelen estar compuestas en torno a 10 personas y en otros países como se citó en el apartado anterior, superan las 20 personas (Amit, Liechtenstein y Prats, 2009; Amit y Liechtenstein, 2009; Casado, Martínez, López, Butler, Sterba, Cazorla y Prado, 2008; Casado, Vidal-Rivas, Brito, Salgado, Verdaguer, Willette y Pastor, 2009). Evidentemente, el tamaño está asociado a la cuantía de patrimonio gestionado, a las actividades desarrolladas, y a la realización de las mismas por personal propio o externo.

Por último, en medio del creciente número de $F O$ y formas híbridas, existe una demanda también creciente de profesionales de élite,

Rivo, E., González-Vázquez, B. y Rodríguez López, N. (2011). Family office: un instrumento de gestión del patrimonio en la Empresa Familiar. Revista de Empresa Familiar, 1(2), 43-57 
dedicados a la gestión del patrimonio, para trabajar en $F O$. Diversos autores analizan las cualidades necesarias para trabajar en una $F O$, incluyendo el deseo de gestionar la dinámica familiar, y gestionar más de un área de especialización (Wolosky, 2002; Avery, 2004; Amit, Liechtenstein y Prats, 2009). Estas cualidades deben combinarse con la capacidad de manejar múltiples complejidades generacionales, numerosas entidades, los objetivos particulares de cada miembro de la familia, y los inevitables problemas emocionales (Bowen, 2004).

Otros artículos abordan cuestiones de mayor relevancia para los profesionales empleados o en busca de trabajo en el nicho de la $F O$ (Newton, 2002; Curtis, 2001; Bowen, 2004; Prince, y File, 1998).

\section{Metodología aplicada: estudio de casos}

Recientemente se han realizado algunos estudios para analizar la implantación de las Family Office y sus consecuencias para la familia empresaria, sobre todo por parte de investigadores norteamericanos (Amit, Liechtenstein y Prats, 2009; Amit y Liechtenstein, 2009). Sin embargo, dada la reciente creación de este tipo de estructuras es difícil encontrar muchas investigaciones sobre ellas, y consecuentemente son muy escasas las referencias a este tema en los trabajos centrados en el estudio de la empresa española.

Sin embargo, a pesar de que la implantación de la $F O$ en España es escasa, se han realizado algunas experiencias muy interesantes que convendría analizar desde el punto de vista organizativo y estratégico. Para realizar esta investigación se utiliza como metodología el análisis de casos. Esta es una herramienta adecuada para este tipo de estudios, ya que se trata de analizar un fenómeno desarrollado en la práctica de la empresa, pero cuyo soporte conceptual no está totalmente establecido. Consecuentemente al evaluar estos ejemplos reales se puede entender la complejidad y las consecuencias de la creación de una $F O$, determinando cuál es la estructura organizativa más interesante que se debe adoptar según los objetivos perseguidos, basándose en la experiencia (Ying, 2003). El estudio de casos es particularmente apropiado para ciertos tipos de problemas en los que la investigación y la teoría se hallan en fases preliminares o iniciales (Roethlisberger, 1977), y para "problemas prácticos delicados donde las experiencias de los participantes son importantes y el contexto de la situación es fundamental" (Bonoma, 1983). Así, antes de que ninguna teoría se formalice, los estudios de casos pueden emplearse para documentar las experiencias que se dan en las empresas (Benbasat, Goldstein y Mead, 1987). Además, el método del estudio del caso permite al investigador responder al "como" y al "por qué", utilizando como punto referencia las opiniones de los actores involucrados (miembros activos del mundo empresarial), a diferencia de las soluciones predeterminadas impuestas por los investigadores (Howorth y Ali, 2001).

Para investigar la $F O$ en España se recurre al análisis de seis casos. En todos ellos se trata de grandes empresas familiares españolas con actividad en distintos sectores económicos y múltiples países.

La selección se ha realizado en función de la clasificación de $F O$ realizada para el caso español, tratando de que exista al menos un ejemplo de cada modelo. Eisenhardt (1989) afirma que la elección aleatoria no es necesaria en la selección de los casos, puesto que el objetivo de la investigación consiste en la elección de casos que puedan replicar o extender una teoría. El objetivo de este tipo de estudio es desarrollar una teoría, no testarla (Eisenhardt y Graebner, 2007). Los investigadores buscan casos críticos para demostrar sus hallazgos principales. Estos casos pueden resultar ser confirmatorios, no confirmatorios, extremos o casos típicos. Los estudios de casos ilustran ideas concretas (Siggelkow, 2007).

El análisis se realiza a través de una serie de entrevistas semiestructuradas realizadas a los máximos responsables de la gestión de la $F O$ correspondiente, con el fin de analizar los logros y las dificultades que está suponiendo la puesta en marcha de la $F O$ para la familia empresaria, 
teniendo en cuenta la reciente creación de todas ellas. Se intentará identificar las características diferenciales en cuanto a estructura, composición, objetivos, órganos de gobierno, y actividades realizadas de cada $F O$.

El objetivo de la investigación se centra en la búsqueda de respuesta a dos cuestiones principales: ¿por qué se crean family offices en España? y ¿cómo se implementan este tipo de estructuras?.

\section{Resultados del estudios de casos}

CASO A: Se trata de un $F O$ que da servicios a la familia sin realizar inversiones propiamente dichas (TIPO 1). La empresa familiar originaria fue fundada en el año 1914, y actualmente está gestionada y controlada por la tercera generación, con un proceso de incorporación de la cuarta generación en calidad de accionistas. En el año 1992 el $F O$ era el director financiero, experimentando una evidente evolución hasta llegar a la estructura actual.

Esta FO tiene como "clientes", diecisiete accionistas, veinte sociedades patrimoniales y treinta familiares no consanguíneos, originando alrededor de cuarenta perfiles distintos de inversión y riesgo.

La estructura de gobierno incluye un Consejo de Administración y un Consejo de Familia.

La $F O$ tiene una vocación de servir de ente aglutinador de la familia. Los servicios prestados se pueden agrupar en siete, siendo los tres primeros los realmente relevantes: gestión de carteras, servicios fiscales, comunicación, información sobre la repercusión mediática del grupo, gestión administrativa, gestión de la flota de vehículos y actividades de formación.

La gestión de carteras se entiende como una gestión de carteras individuales de cada accionista o de cada grupo familia, pero no como inversiones comunes. El servicio se basa en tres ejes: uno es la protección del capital mediante estructuras eficientes; segundo, el crecimiento o la preservación de capital, definición del perfil de riesgo de cada inversor, selección de gestores, selección de productos, para acabar estableciendo las carteras de inversión de cada uno (para ello se cuenta con un equipo de tres personas que provienen del sector del mercado y dedican gran parte de su tiempo a estas tareas). Y tercero, control; seleccionando los custodios en base a unos criterios de calidad, realizando el seguimiento y, sobre todo, un reporting consolidado, cuestión importante a efectos de transparencia. Los accionistas disponen semanalmente de una cartera actualizada. Las carteras financieras pueden incluir inmuebles y participaciones empresariales.

La actividad de formación es especialmente relevante ante la próxima incorporación de la cuarta generación; la familia quiere que se incorporen no sólo como accionistas, sino como buenos accionistas. Se organizan uno o dos seminarios de formación al año.

El equipo de la $F O$ consta de catorce personas, nueve profesionales y cinco staff. Para no cargar demasiado la estructura, se apoyan mucho en profesionales externos en algunos procesos. Existe una tendencia clara hacia la externalización.

La $F O$ se plantea como retos de futuro o cuestiones a resolver la incorporación de la cuarta generación como buen accionista; la tarificación de servicios (¿quién paga la gestión de la FO?, ¿se paga en función de la participación, según el uso, o en función del volumen de patrimonio?), y la externalización de actividades.

CASO B: Se trata de una $F O$ que no sólo presta servicios a la familia, sino que además realiza actividades de inversión (TIPO 2). Su objetivo fundamental es la preservación de la riqueza. La empresa familiar asume el mayor riesgo, siendo la generadora de riqueza, mientras que la $F O$ trata de conservar el patrimonio, asumiendo menor riesgo y una mayor diversificación.

La FO responde a una formulación estratégica de la familia, que en un momento de liquidez importante, decide separar determinados activos patrimoniales del negocio troncal. Los miembros fundadores de la segunda generación, ofrecen de manera generosa a los miembros de la tercera generación, decidir entre 
dos alternativas: tener los activos separados individualmente por cada uno de ellos y gestionarlos libremente, o tener esos activos integrados y gestionados mediante una oficina más profesionalizada que aunara intereses financieros y gestión de activos más una serie de servicios.

Las ventajas que identificó la tercera generación fueron: economías de escala en la negociación con entidades financieras; acceso a muy buenos servicios con tratamientos preferenciales y con un track record mucho más seguido; entrada en productos financieros de difícil acceso si no se posee una masa crítica necesaria o la influencia necesaria; la posibilidad de abordar proyectos difíciles a nivel individual; disminución del riesgo al poder diversificar una masa mayor; y sobre todo, disminuir cargas fiscales al no realizar separaciones patrimoniales constantes, mucho más caras y difíciles.

Existe un Consejo de Familia donde además de los miembros familiares, van a participar los miembros no familiares con capacidad de decisión y de voto. Se requiere una mayoría de dos tercios para aprobar un acuerdo. Ninguna rama familiar sola puede tomar una decisión determinada. Se plantea la búsqueda sistemática del consenso de todos tratando de encontrar la resolución de un conflicto. Este Consejo se reúne cuatro veces al año, y cuenta con tres miembros consejeros independientes no familiares. El Consejo busca básicamente alcanzar el consenso; está formado por el Comité de Inversiones, y el Comité de Planificación y Estrategia. Entre sus competencias están la de establecer las reglas de gobierno, implantación de procedimientos, programas de seguros, planes de pensiones, planes de carrera, filantropía, coaching, proyectos de remuneraciones y planes de incentivos para empleados. Se establece una remuneración por pertenecer al Consejo, no muy elevada, pero que ayuda a que los miembros de la familia se sientan más involucrados.

Otro órgano de gobierno es el Consejo de Propietarios, que agrupa a los accionistas antes de entrar, y sindicando un poco según el tipo de pactos y acuerdos, para formar parte de los consejos, las juntas generales, etc.

La dirección del $F O$, cuenta con comités ejecutivos, agrupados en dos grandes bloques. Uno, incluye los responsables de administración, coordinación y gestión económica de las actividades, y el segundo incorpora los responsables de las áreas específicas de negocio (el área inmobiliaria, el área hotelera y gestión patrimonial; el área financiera; el área de private equity, sobre todo en venture capital, y actividades empresariales varias).

Esta organización implica la necesidad de un perfil de personal profesional y muy autosuficiente, con mucha experiencia polivalente.

La $F O$ realiza actividades en diversas áreas: inmobiliaria (residencia, oficinas y comercial), hotelera (gestión de hoteles urbanos en propiedad), financiera (gestionada por una consultoría totalmente externa), private equity (participa en empresas que no cotizan en Bolsa; se invierte por razones de diversificación de riesgo, rentabilidad esperada y ventajas fiscales), y nuevos proyectos en el sector salud y healthcare.

En cuanto a administración y servicios, se prestan servicios de en asesoría financiera, inmobiliaria, fiscal, jurídica, laboral y servicios generales tanto a miembros de la familia consanguínea como a miembros de la familia política. Se fomenta mucho la formación, tanto interna como externa, y realizan workshops que puedan servir a miembros de la familia de una u otra generación (que no necesariamente tienen que ser los más jóvenes).

Se creó un portal familiar con una web con accesos específicos a cada uno de los miembros, para poder consultar su reporting, con bibliografía, todos los resúmenes de las actividades, intercambio de álbumes de fotos, gacetas familiares, etc...

CASO C: Se trata de una FO del TIPO 2. El origen de los fondos comienza en 1999 con la salida a Bolsa de la compañía. A partir del año 2000 se empieza a trabajar en la creación de la $F O$. En el año 2002 el capital gestionado 
superaba el billón de euros, pero con una estructura muy incipiente, basada en los orígenes anteriores: el fundador administraba un patrimonio considerable de forma muy personal.

La FO responde ante un Consejo de Familia, presidido por un independiente externo en el que participan cinco miembros de la familia $\left(2^{\mathrm{a}}\right.$ generación).

El director de la $F O$ responde ante el Consejo de Administración de la $F O$ del cual es consejero delegado, y al que pertenecen cuatro miembros de la familia, un consejero secretario y tres asesores externos (tres directores de $F O$, uno canadiense y dos europeos).

Podríamos distinguir entre Family Office /servicios y Family Office/inversiones. En este último caso se decidió realizar una allocation central y estratégica de acuerdo al perfil de la familia y al origen de los fondos. Como la familia ya asume suficientes riesgos en la empresa familiar, la inversión gestionada a través de la $F O$ es mucho más conservadora (la $F O$ gestiona la cartera de los activos tradicionales a través de gestores externos; aproximadamente se trabaja con unos 50 gestores). Se externalizan al máximo todos los procesos, tanto a nivel de sociedad gestora como departamento de control, administración de carteras, y cálculo de valor liquidativo.

En cuanto a la estructura propia, la $F O$ está compuesta por 22 personas: seis personas en contabilidad, dos secretarias, cuatro abogados, una persona en servicios generales, dos informáticos, dos personas en back office, cuatro personas dedicadas a inversiones y una persona que lleva toda la estrategia de derivados. No hay nadie de la familia trabajando en la $F O$.

A finales del 2008 la composición de las carteras era $42 \%$ en equity, $20 \%$ en renta fija, 9\% en fondos de inversión, y $29 \%$ en private equity $\mathrm{y}$ activos inmobiliarios.

La diversificación es un objetivo crítico para esta familia, que sigue asumiendo altas dosis de riesgo con las compañías cotizadas gestionadas.

CASO D: Se trata de una $F O$ del TIPO 3, creada con el objetivo de diversificación.
La empresa familiar fue creada en el año 1946. Tras el fallecimiento del gestor de la $3^{\mathrm{a}}$ generación, asume la gestión un miembro de la $4^{\text {a }}$ generación, creando la $F O$. Ésta no está pensada para dar servicio a nivel particular a los miembros de la familia ni para actuar como vehículo de gestión patrimonial individual de los miembros de la familia; está configurada como una sociedad donde se concentran las actividades empresariales del grupo.

Los activos individuales de los miembros de la familia y los activos de la empresa comunes se gestionan con estructuras separadas bajo criterios de riesgo diferentes y con equipos diferentes. La familia tiene claro en dividir, por un lado, lo que serían los activos propios personales que se gestionan desde un punto de vista de riesgo muy controlado, de preservación de patrimonio y por otro lado, lo que serían los activos propiamente empresariales.

Uno de los aspectos singulares del grupo es su cotización en Bolsa. Esa situación les obliga a ser muy rigurosos con la gestión y muy transparentes. También les proporciona liquidez; liquidez que pueda ser necesaria si algún miembro de la familia decide desinvertir parte de su patrimonio, y que permite dar entrada a grupos inversores afines. De hecho, parte del accionariado lo conFOrman grupos familiares que co-invierten con la familia; utilizan la plataforma de esta $F O$ como coinversor aprovechando su knowhow. Uno de los objetivos fijados en el 2006 era dar los pasos necesarios para dar el salto al mercado continuo. Dado el contexto de mercado y la coyuntura se ha decidido aplazar el mismo, sin olvidarlo.

En cuanto a la estructura de los órganos de gobierno, el Consejo de administración de la empresa está conformado por 9 personas, 4 miembros de la familia y 5 independientes. Existe un Comité de dirección donde también la mayoría son ejecutivos, y un Comité inmobiliario que se reúne mensualmente con mayoría de ejecutivos. En el Comité inversor está la consejero delegado, miembro de la $4^{\mathrm{a}}$ generación, el director de la $F O$ y un consejero independiente; tres personas que toman las decisiones de inversión más importantes que se 
tienen que elevar al Consejo por su singularidad.

El grupo se estructura en dos áreas de negocio separadas en cuanto a organización y estructura. Por un lado, la actividad más empresarial (el área inmobiliaria) que representa alrededor del $40 \%$ del valor de mercado del grupo. Por otro lado, el área de inversiones que representa un $60 \%$.

En la parte inmobiliaria cuentan con dos subdivisiones, una parte de promoción, de tamaño mediano, conservadora en la gestión. Una segunda subdivisión es la parte de arrendamientos.

En el área de inversiones se diferencian respecto a otras estructuras u organizaciones. Se analizan las inversiones desde el punto de vista de empresario. Se intenta conocer el negocio y se compran participaciones en el mismo. No actúan como inversores estrictamente financieros. Se trata de un enfoque de inversión de medio y largo plazo (no oportunista ni especulador). Se plantea un objetivo de rentabilidad de alrededor del $15 \%$; que puede variar en función de la tipología del negocio.

De forma general, sólo se hace gestión directa. La $F O$ cuenta con un equipo de analistas propios, formado por 8 personas. No se externaliza la gestión, ni tampoco se invierte en vehículos de inversión colectiva.

La estructura del área de inversiones se subdivide en tres partes. La primera de participaciones estratégicas, que representan alrededor del $75 \%$ del valor de la cartera de inversiones. La segunda es la cartera de Bolsa y la tercera, en fase de desarrollo, son las inversiones en capital riesgo o en empresas no cotizadas.

Respecto al organigrama de profesionales, hay 30 personas en el área inmobiliaria, 10 en el área de control y gestión administrativa y 8 en el área de inversión.

CASO E: $F O$ del TIPO 3 con origen en la venta de la empresa familiar.

Creada en el año 1998 para gestionar el patrimonio líquido generado tras la venta de la empresa familiar (con diez años de historia tiene una de las trayectorias más largas de las $F O$ implantados en España) sigue un modelo que se aproxima más a una sociedad de inversión que a una sociedad que da servicio a los distintos miembros de una familia que comparten negocios.

Posee la estructura de una sociedad limitada con administradora única. No tiene Consejo de Administración, sino un ejecutivo principal.

La familia se involucra mucho, sigue muy de cerca la compañía pero al mismo tiempo delega mucho en los profesionales. Dentro de la FO uno de los ejecutivos es el hijo de la principal accionista que toma una participación activa en la gestión de la compañía.

Poseen una estructura ajustada con gente de perfiles en general senior. La gestión interna es muy sencilla al no dar servicios a la familia (que se encuentra en primera generación). Las decisiones tienden a ser bastante personalistas, existiendo pocos comités.

La gestión de esta $F O$ se basa en la diversificación y la liquidez. Incluye una cartera con activos inmobiliarios, fondos de inversión, renta variable cotizada, renta variable privada; estructura similar a las $F O$ estadounidenses. Existe una sobreponderación en renta variable cotizada y en fondos de inversión, frente a las típicas $F O$ europeas.

La actividad principal de esta $F O$ se centra en la gestión de los fondos de inversión. Es la actividad a la que dedican más medios (ejecutivo por volumen gestionado), y donde el dinero no es todo de la familia (aunque ostenta la mayoría de la inversión en estos fondos); se da entrada a inversores afines o que tienen objetivos de inversión similares.

CASO F: $F O$ del TIPO 3 con origen en la venta de la empresa familiar.

Creada en el año 2006 tras la venta de la empresa familiar que se encontraba en $3^{\text {a }}$ generación.

En cuanto a la gestión de la $F O$, para esta familia el patrimonio gestionado no incluye sólo activos financieros, inmuebles y otras inversiones, sino que incorpora bienes de la familia, sus relaciones, su proyección social, sus 
habilidades diferenciales, etc. Todo ello forma parte de los "activos" de la familia que considera que el dinero no es lo que la mantiene unida a largo plazo; es el legado de principios y valores lo que va a unir a la familia a lo largo de las generaciones.

Consecuentemente, el objetivo de la $F O$ es la satisfacción de la familia, concepto que incluye la satisfacción de vivir en familia. De forma que no sólo hablamos de financiar su nivel de vida a lo largo de su vida y satisfacer sus deseos filantrópicos entre otros factores sino sobre todo del placer de vivir en familia.

Dentro del perímetro de gestión de la empresa, los comités de Dirección del Grupo y de la Fundación reportan al Consejo de Dirección y éste a la propiedad. Además de estos órganos de gestión "del negocio", existe un comité especial para gestionar las sinergias entre la fundación y el grupo.

A nivel familiar, el máximo órgano es el Consejo de familia, integrado por miembros de la familia y externos. Entre sus funciones está la gestión del sistema de cohesión familiar.

Un aspecto relevante de esta $F O$ es que cada rama familiar es libre de decidir sus inversiones. La $F O$ se organiza matricialmente. Hay unos responsables de cada uno de los proyectos y, luego, hay una serie de servicios comunes a todos ellos como la contabilidad, los estudios, el análisis de proyectos, etc... Los seguimientos se realizan a nivel proyecto y a nivel función. Como la $F O$ sólo está compuesta por 10 personas se logra flexibilidad y polivalencia.

En la Tabla 2 se resumen las principales características de las $F O$ analizadas, identificando el tipo de $F O$, el objetivo perseguido con la $F O$, las actividades realizadas por la $F O$, el personal contratado para la realización de las mismas (indicando si se recurre o no a la externalización), así como los órganos de gobierno implantados para la gestión y control de la $F O$.

\section{Conclusiones}

Acorde con los objetivos planteados, en este trabajo hemos profundizado en el concepto de family office, así como en las principales motivaciones que llevan a su creación. En este sentido, el planteamiento para crear una $F O$ suele hacerse a partir de grandes patrimonios (superiores a los 25 millones de euros en España, y más de 65 millones de euros en otros países) dado su elevado coste operativo.

Se considera que las razones más importantes para su creación se basan en la privacidad, el control, la flexibilidad y el servicio individualizado.

Asimismo, se han descrito las diversas clasificaciones de $F O$ existentes, resaltando la simplicidad e idoneidad de la clasificación utilizada en España en la que se distinguen sólo 3 tipos. La clasificación de las $F O$ se realiza en base a los objetivos perseguidos y en consecuencia en base a las actividades realizadas por la misma. Pese a la uniformidad de clasificaciones, es de destacar que la composición de la $F O$ puede oscilar mucho según se adopte una estrategia de realización de las actividades interna o externa.

Existen $F O$ que prestan servicios a la familia, sin realizar inversiones propiamente dichas, como es el CASO A analizado (tipo 1). En otros casos, la familia decide que hay un patrimonio a gestionar, habilitándose una estructura dedicada exclusivamente a la gestión, realizando inversiones para obtener la máxima rentabilidad al patrimonio, sin prestar servicios a la familia (tipo 3). Este tipo de $F O$ puede tener su origen en la desinversión familiar (CASOS E y F), o en la búsqueda de diversificación del patrimonio, CASO D. Finalmente, existe un $F O$ mixto, que presta servicios a la familia, y al mismo tiempo actúa como entidad inversora (CASOS B y C); se trata de la categoría más completa (tipo 2).

El número de $F O$ implantado en España es reducido, pero cada vez hay una mayor tendencia a interesarse por este tipo de estructuras, al considerarlas como las más adecuadas a la hora de gestionar el patrimonio familiar $^{2}$. En esta línea, la investigación

\footnotetext{
${ }^{2}$ Sólo el 15 de las 100 mayores empresas familiares que componen el Instituto de la Empresa Familiar poseen en la actualidad una FO estructurada (información facilitada por el Instituto de la Empresa Familiar).
} 
preliminar (Martiros, y Millay, 2006) sugiere que la $F O$ está buscando foros de conocimiento que proporcionen una normalización de su gestión así como más transparencia de mercado, a fin de garantizar una experiencia más coherente y eficaz.

En el estudio de casos realizado en este trabajo de investigación se observa, que con independencia de la modalidad de family office, hay una tendencia generalizada a la externalización de parte de las actividades realizadas. Tendencia que sucede a nivel mundial como queda reflejado en el estudio realizado por el IESE (Amit yLiechtenstein, 2009), observándose que es mayor en el caso norteamericano que en el caso europeo.

En cuanto a la estructura de gobierno se observa en, con carácter general que todas las empresas que cuentan con una family office implementada, tienen en funcionamiento un Consejo de Administración, un Consejo de Familia, y en varios casos, diversos Comités Ejecutivos. En cuanto a las actividades realizadas, éstas son de diversa índole, aunque suelen confluir las inversiones en el área inmobiliaria, en private equity, en renta variable cotizada, así como en inversiones directas en empresas no cotizadas.

Dados los resultados obtenidos se plantea como futura línea de investigación la evaluación del rendimiento de la $F O$ desde dos perspectivas: la influencia de la estructura de gobierno de la $F O$ sobre sus resultados, y la influencia de la externalización de actividades sobre el rendimiento de la $F O$. Ambas serán objeto de estudios posteriores.

\section{Bibliografía}

Allen, C. (2007). The Changing Face of the Family Office. Global Investor, 200, 21-22.

Amit, R. y Liechtenstein, H. (November 2009). Report Highlights for "Benchmarking the Single Family Office: Identifying the Performance Drivers". Wharton University.

Amit, R. (2006). Family Offices in the US. Working document.
Amit, R., Liechtenstein, H., y Prats, M.J. (2009). Single Family Office: Private Wealth Management in the Family Context. Wharton University.

Avery, H. (2004). Keeping It in the Family. Euromoney, 35, 236-246.

Bensabat,I., Goldstein, D.K., y Mead, M. (1987). The Case Research Strategy in Studies of Information Systems. Mis Quarterly, 11, 369386.

Bonoma, T.V. (1983). A Case Study in Case Research: Marketing Implementation. Working Paper, 9, 585-142, Harvard University Graduate School of Business Administration, Boston, MA.

Bowen, J. (2004). In the Family Way. Financial Planning, 8, 31-33.

Casado, F., Martínez, J.L., López, F., Butler, C., Sterba, A., Cazorla, J., y Prado, D. (2008, junio). Family Office en la empresa familiar. Documento 147, Instituto de la Empresa Familiar, Barcelona.

Casado, F., Vidal-Rivas, E., Brito, A., Salgado, A., Verdaguer, J., Willette, R., y Pastor, E. (2009, junio). Family Office en la empresa familiar. Documento 152, Instituto de la Empresa Familiar, Barcelona.

Curtis, G. (2001). Establishing a Family Office: A Few Basics.. Greycourt White Paper, 10.

Eisenhardt, K.M. (1989). Building theories from case study research. Academy of Management Review, 14(4), 532-550.

Eisenhardt, K.M., y Graebner, M.E. (2007). Theory building from cases: Opportunities and challenges. Academy of Management Journal, 50(1), 25-32.

Gray, L. (2005). How Family Dynamics Influence the Structure of the Family Office. Journal of Wealth Management, 8(2), 9-17.

Gray, S. (2004). Changing Face of the Family Office. International Money Marketing, 23-23.

Howorth, C., y Ali, Z.A. (2001). Family business succession in Portugal: An examination of case studies in the furniture 
industry. Family Business Review, 14(3), 231- Roethlisberger, F.J. (1977). The Elusive 244.

Phenomena. Harvard Business School, Division

Martin, H.F., Lansberg, I., y Darst, D. (1999, of Research. Boston, MA.

noviembre). Las oficinas familiares: la Shaw-Grove, H., y Prince, R.A. (2004). Family experiencia norteamericana. Documento 104, Offices: Assets and Motivacions. Financial Instituto de la empresa familiar, Barcelona.

Advisor Magazine, October.

Martiros, S., y Millay, T. (2006). A Framework for Understanding Family Office Trends. White Paper.

Siggelkow, N. (2007). Persuasion with case studies. Academy of Management Journal, 50(1), 20-24.

Newton, C. (2002). Adopting the Family Office. Journal of Financial Planning, 15(6), 66-74

Wolosky, H. (2002). Family Offices Come Downtown. Practical Accountant, 35(3), 23-27.

Prince, R.A., y File, K.M. (1998). All in the Family Office. Financial Planning, 28(10),153.

Rivo López, E., Rodríguez López, N., González Ying, R. (2003). Case Study Research: Design and Methods. Thousand Oaks, CA: Sage Publications ( $3^{\mathrm{a} e d}$.).

Vázquez B. (2011, septiembre). La family office en España: un estudio de casos, XXI Congreso nacional de ACEDE, Barcelona. 